\title{
Arrival time of halo coronal mass ejections in the vicinity of the Earth ${ }^{\star}$
}

\author{
G. Michałek ${ }^{1,3}$, N. Gopalswamy ${ }^{2}$, A. Lara ${ }^{3,4}$, and P. K. Manoharan ${ }^{3}$ \\ 1 Astronomical Observatory of Jagiellonian University, Cracow, Poland \\ e-mail: michalek@oa.uj .edu.pl \\ 2 NASA Goddard Space Flight Center, Greenbelt, MD 20771, USA \\ 3 The Catholic University of America, Washington DC 20064, USA \\ ${ }^{4}$ Instituto de Geofísica, UNAM, México
}

Received 2 February 2004 / Accepted 26 April 2004

\begin{abstract}
We describe an empirical model to predict the 1-AU arrival time of halo CMEs. This model is based on the effective acceleration described by Gopalswamy et al. (2000a, Geophys. Res. Lett., 27, 145). We verify the Helios/Pioneer Venus Orbiter(PVO) estimation of the effective acceleration profile (Gopalswamy et al. 2001a, J. Geophys. Res., 106, 29207) by considering all full halo CMEs recorded by SOHO/LASCO coronagraphs until the end of 2002. In comparison with previous studies, the present work includes CMEs of a wider range of initial velocities. To improve the accuracy of prediction, we propose to introduce the effective acceleration from two groups of CMEs only, which are expected to have no acceleration cessation at any place between the Sun and Earth. In addition, we consider acceleration cessation distance dependent on initial velocities of a given event CME. For a detailed analysis of this model, we examine projected sky-plane and space speeds (Michałek et al. 2003, ApJ, 584, 472) of CMEs. We show that a correct acceleration profile is crucial for the estimation of 1 AU arrival time of halo CMEs. We estimate that the CME arrival times can be predicted with an average error of 9 and $11 \mathrm{~h}$ for space and sky-plane initial velocities, respectively.
\end{abstract}

Key words. Sun: coronal mass ejections (CMEs)

\section{Introduction}

Space Weather is significantly controlled by coronal mass ejections (CMEs) which can affect the Earth in many ways. CMEs originating close to the central meridian of the Sun, directed toward the Earth, are of the biggest scientific concern. For space weather forecasts it is important to know when a solar disturbance would reach the Earth. CMEs are ejected and accelerated by the magnetic field of the corona. During travel through space this magnetic force decreases and other processes may accelerate CMEs. They interact with solar wind and other events (Gopalswamy et al. 2001c, 2002, 2003b; Manoharan et al. 2004). Fast CMEs are decelerated mostly by solar wind due to friction which is proportional to the square ofthe velocity difference. During a maximum of solar activity we expect to observe many CME interactions. CMEs can be suddenly accelerated or can merge into another event. This mechanism is unpredictable.

The early models concerning the arrival of interplanetary (IP) shock were based on observations of metric type II radio bursts (Smart \& Shea 1985; Smith \& Dryer 1990). In these models, the drift rate of type II bursts was used to

$\star$ Table 1 is only available in electronic form at http://www. edpsciences.org determine the speed of the shock and to develop a scheme to predict their appearance in the vicinity of the Earth. However, it was pointed out by Gopalswamy et al. $(1998,2001 b)$ that there is little connection between coronal shocks (inferred from metric type II bursts) and the IP shocks (detected in situ by spacecraft). Furthermore, IP shocks are not always followed by interplanetary CMEs (ICMEs), which are responsible for geomagnetic storms (Cane et al. 2000, 2003). For example, IP shocks observed in situ without ICMEs are caused by large limb CME (Gopalswamy et al. 2001b). Therefore, predicting CME arrival at $1 \mathrm{AU}$ is of fundamental importance (Gopalswamy et al. 2001b) and can be extended to predict shock arrival (Gopalswamy et al. 2003a,b).

Combining CME observations made by SOHO/LASCO and ICMEs measurements near the Earth, Gopalswamy et al. (2000, 2001a) developed an empirical model to predict the 1-AU arrival time of CMEs. The model was based on the fact that the speed distribution of ICMEs, detected by the Wind spacecraft, was much narrower (in the range $350-650 \mathrm{~km} \mathrm{~s}^{-1}$ ) in comparison to the velocity distribution of CMEs observed by SOHO/LASCO near the Sun (in the range 150-1050 $\mathrm{km} \mathrm{s}^{-1}$ ). They postulated that CMEs undergo an effective acceleration due to interaction with the solar wind. This effective acceleration was assumed to be constant over the Sun-Earth distance 
and was defined as the difference between the initial $(u)$ and final $(v)$ speeds divided by the time $(t)$ taken by a given CME to reach Earth. They found a definite correlation between the effective acceleration ( $a$ ) and $u: a=1.41-0.0035 u$ ( $a$ and $u$ are in units $\mathrm{m} / \mathrm{s}^{2}$ and $\mathrm{km} \mathrm{s}^{-1}$ respectively; Gopalswamy et al. 2000a). SOHO/LASCO measurements of Earth-directed CMEs are subject to major projection effects (Gopalswamy 2000b). To minimize the projection effects, Gopalswamy et al. (2001a, hereafter Paper I) used archival data from spacecraft in quadrature to get an improved effective acceleration model: $a=$ $2.193-0.0054 u$. These relations can be used in the kinematic equation, $S=u t+a t^{2} / 2$, where $S$ is the distance travelled by the CME, to predict the arrival time at 1-AU. It is important to note that the only free parameter required by the model is the initial CME velocity. To generalize the model, Gopalswamy et al. (2001a) assumed that the effective acceleration can cease at some distance $(d)$ between the Sun and Earth. Best results were obtained when the acceleration ceased at a distance of 0.76 AU. With this model, Gopalswamy et al. (2001a) were able to predict the travel time within an error of $10.7 \mathrm{~h}$. They found that a simple geometrical correction (Leblanc et al. 2001) for projection effects did not work and suggested that such effects were partly compensated by CME expansion.

Recently Michałek et al. (2003) developed a new method to obtain the space-speed of CMEs, which minimizes the projection effects for full halo CMEs and, therefore, gives a better approximation for the CME initial speed. In this work we have obtained the space speed of Earth directed full halo CMEs recorded by the SOHO/LASCO coronagraph from January 1996 to December 2002. In comparison with previous studies, the present work includes a greater number of CMEs from a longer period of solar activity. To improve the accuracy of prediction, we propose to introduce the effective acceleration from two groups of CMEs only, which are expected to have no acceleration cessation at any place between the Sun and Earth. In addition, we consider the acceleration cessation distance to be dependent on initial velocities of a given event CME. To obtain a detailed analysis of this model, we examine projected sky-plane and space speeds (Michałek et al. 2003) of CMEs. Then we looked for the ICME counterparts and compute the acceleration profile. Our data set is expanded not only in number but also in the velocity interval considered in previous works. In this paper we consider only front-sided halo CMEs (FHCME)

\section{Data}

To build an empirical model to predict 1-AU arrival time we follow the same steps as in Gopalswamy et al. (2000a): (1) select the FHCME-ICME pairs (in this case we started with all FHCMEs observed by LASCO and found the related ICMEs); (2) determine an empirical relation between the effective acceleration and the initial speed of CMEs (using a new method to obtain the initial space-speed); and (3) use the kinematic equations to obtain the transit time $t$ (using different criteria to select the cessation distance). We consider all CMEs (width $=360^{\circ}$ from 1996 until the end of 2002 as listed in http://cdaw.gsfc.nasa.gov/CME_list
In situ counterparts of front-sided HCMEs can be recognized in the magnetic field and plasma measurements as ejecta (EJs) or magnetic clouds (MCs). Magnetic clouds have the following characteristic properties: (1) the magnetic field strength is higher than the average; (2) the proton temperature is lower than the average; and ( 3 ) the magnetic filed direction rotates smoothly (Burlaga 1988, 2002, 2003a,b; Lepping et al. 1990). In this paper we refer to both MCs and EJs as interplanetary CMEs (ICMEs). The presence of these signatures changes from one ICME to an other. The ICME boundaries can be fuzzy and the arrival time of MCs is determined with some error. This is also the reason that in our considerations the average speeds of ICMEs are applied.

Using the SOHO/LASCO catalog and Wind spacecraft data, we were able to select 83 FHCME-ICME pairs covering a period of time from the beginning of 1996 until the end of 2002. The total number of FHCMEs during this period is 123 . Note that the total number of halo CMEs reported by Gopalswamy et al. (2003) during this period is 468 , which included all CMEs with width $>270^{\circ}$ irrespective of whether they are frontsided or back-sided. Events studied in this paper are shown in Table 1. The first three columns are from the SOHO/LASCO catalog (date, time of first appearance in the coronagraph and projected speed). Details about the SOHO/LASCO catalog and method of measurements is described by Yashiro et al. (2003).

Assuming that a HCME has a constant velocity near the Sun and a cone-shaped structure, Michałek et al. (2003) were able to obtain the space speed, which is different from the one obtained by correcting for geometrical projection. This technique requires measurements of sky-plane speeds and the times of first appearance of the halo CMEs above the opposite limbs. We apply this technique to obtain the parameters of all the HCMEs. The space velocities could be determined for 49 HCME-ICME pairs and are shown in Col. 4 of Table 1. For the remaining 34 halo CMEs, it was not possible to obtain the space speed. This situation occurs when a HCME is too faint to generate the height-time plot at opposite limbs or when a HCME is symmetric. It is important to note that for some events, the space velocity determined by this technique could be smaller than the projected speeds reported in the LASCO catalog. This is because the Michałek et al. (2003) technique applies only to the beginning phase of CMEs, whereas the CME catalog gives average speeds within the LASCO C2 field of view. When a CME strongly accelerates, the sky plane velocity can increase significantly in the LASCO field of view. By examining solar wind plasma data from the Solar Wind Experiment (Wind/SWE) http ://web.mit . edu/space/www/wind/) instrument and interplanetary magnetic field data (from Magnetic Field Investigation, http://lepmfi.gsfc.nasa.gov/mfi), we identified interplanetary CMEs. For each event, we determine the approximate start time and average speed of the ICME. The date and average speed of 83 ICMEs are presented in Cols. 6 and 7 of Table 1. The travel time (Col. 5), $t=T_{\mathrm{ICME}}-T_{\mathrm{HCME}}$, was obtained from the difference in the time of first appearance of a given CME in the LASCO coronagraph $\left(T_{\mathrm{HCME}}\right)$ and the respective ICME in Wind observations $\left(T_{\mathrm{ICME}}\right)$. 

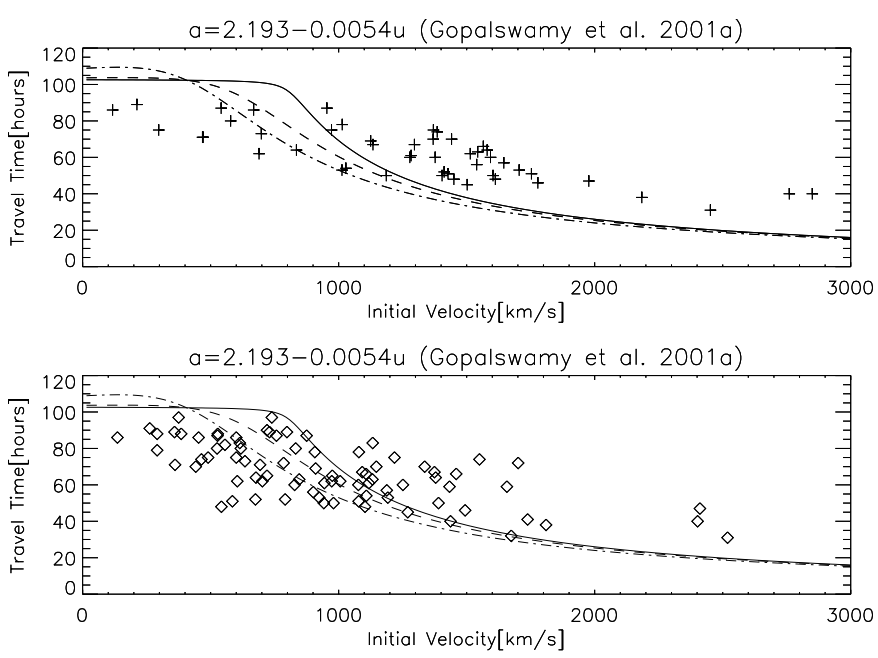

Fig. 1. Comparison between predicted and observed travel times based on the acceleration profile $a=2.193-0.0054 u$ (Gopalswamy et al. 2001a). The solid, dashed and dot-dashed lines represent model predictions for acceleration-cessation distance equal to 1.0, 0.75 and $0.5 \mathrm{AU}$, respectively. The plus symbols denote the data points corrected for projection effect (49 HCME-ICME pairs, upper panel) and diamond symbols for data points uncorrected for the projection effect (83 HCME-ICME pairs, bottom panel).

\section{Analysis}

In Fig. 1 we plot the observed travel times for both sets: the 49 corrected (plus symbols, upper panel) and 83 uncorrected (diamonds, lower panel) CMEs considered in this study. For comparison, we have also plotted the predicted travel times computed based on the acceleration profile of Paper I:

$a=2.193-0.0054 u$,

at three different acceleration cessation distances $d=$ 0.50, 0.75, 1.00 AU. The model works well for events with initial velocities in a range between $700 \mathrm{~km} \mathrm{~s}^{-1}$ and $1200 \mathrm{~km} \mathrm{~s}^{-1}$. Slower and faster events fall below and above the prediction curves. The same tendency is observed for data points with corrected and uncorrected velocities. This indicates that slow events move faster and fast events move slower than predicted in Paper I. This means that the effective acceleration for slow CMEs and deceleration for fast CMEs must be more efficient than those given in Paper I.

To improve the travel time prediction we derive a new acceleration profile using corrected space speeds of 49 halo CMEs in an expanded speed range (from 117 to $2850 \mathrm{~km} \mathrm{~s}^{-1}$ ). Figure 2 (left panel) shows the effective acceleration computed from the observed transit times and the initial (white-light) and final (in situ) speeds, versus the initial space speed for 49 FHCME-ICME pairs listed in Table 1. The correlation between acceleration and initial velocity is very good (the correlation coefficient is 0.94 ).

\subsection{Second order fit}

The solid line in Fig. 2 (the left panel) represents a quadratic fit to the data points, giving an effective acceleration:

$a_{n 2}=0.28+5.3 \times 10^{-4} u-2.1 \times 10^{-6} u^{2}$.

The effective acceleration almost vanishes for slow events and is less efficient for fast events than the quadratic fit given in Paper I:

$a_{o 2}=0.74+8.6 \times 10^{-4} u-4.08 \times 10^{-6} u^{2}$.

The travel time obtained using the acceleration given by Eq. (2) is shown in Fig. 3. The observed travel times for corrected (upper panel) and uncorrected (bottom panel) velocities are shown by the data points. We consider the prediction curves corresponding to the acceleration cessation distance equal to 0.50 , 0.75 and $1 \mathrm{AU}$, dot-dashed, dashed and solid lines, respectively. For fast events, CMEs generally arrive later than the model prediction and for slow events, the effective acceleration is not sufficient to push CMEs substantially ahead, so predicted travel times for these events are longer than the observed times.

\subsection{First order fit}

The dashed line in Fig. 2 (the left panel) is the linear fit to data points:

$a_{n 1}=4.11-0.0063 u$.

The coefficients of this relation are different from the Gopalswamy et al. (2000) models, probably due to the inclusion of several fast events, which determine the slope of the linear fit.

The travel times computed using the acceleration profiles given by Eq. (4) are plotted in Fig. 4, the comparison between predicted and observed travel times for corrected (upper panel) and uncorrected (bottom panel) velocities are shown. The prediction curves corresponding to the acceleration cessation distances of $0.50,0.75$ and $1 \mathrm{AU}$ together with measured points are presented in the figure. Again the agreement with observations is not good. In this case, CMEs seem to arrive later than the model predictions.

\subsection{New possibilities}

In Sects. 3.1 and 3.2 we demonstrated that acceleration profiles determined using the method presented in Paper I cannot give good fits to observations. In the present subsection we propose a new approach in determining acceleration profiles. The differences between observations and model predictions might be caused by the fact that we do not know where (or when) the acceleration ceases. To overcome this problem we explore two possibilities: i) compute a model using only two extreme cases (when there is no acceleration and when the acceleration still acting at 1-AU); and ii) assuming that the acceleration ceases when each CME reaches a speed equal to the mean solar wind speed $\left(\sim 450 \mathrm{~km} \mathrm{~s}^{-1}\right)$. 

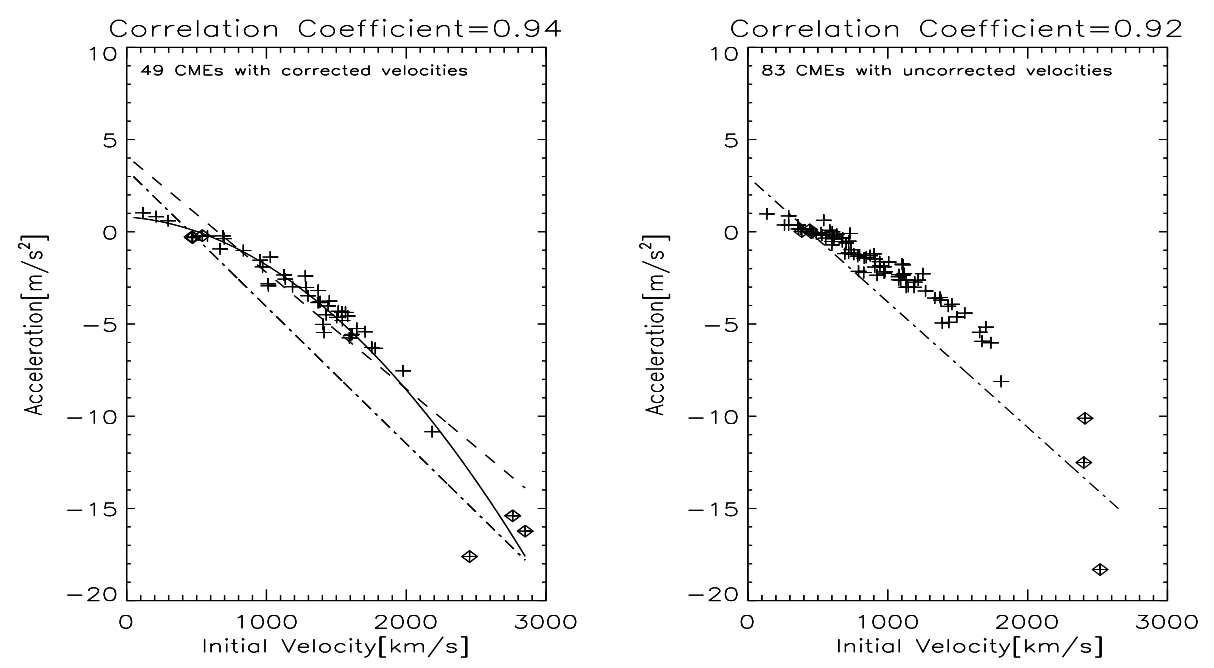

Fig. 2. The effective acceleration profiles versus initial speed of HCMEs listed in Table 1. The plus symbols denote the data points. The left panel show 49 HCMEs with corrected initial velocities and the right 83 CMEs with uncorrected initial velocities. The solid and dashed lines (in the left panel) are quadratic and linear fits to the data points respectively. The dot-dashed lines (in the both panels) are linear fits for the three events with initial velocity closest to $450 \mathrm{~km} \mathrm{~s}^{-1}$ and the three fastest events (data points marked additionally by diamonds) from the entire sample.
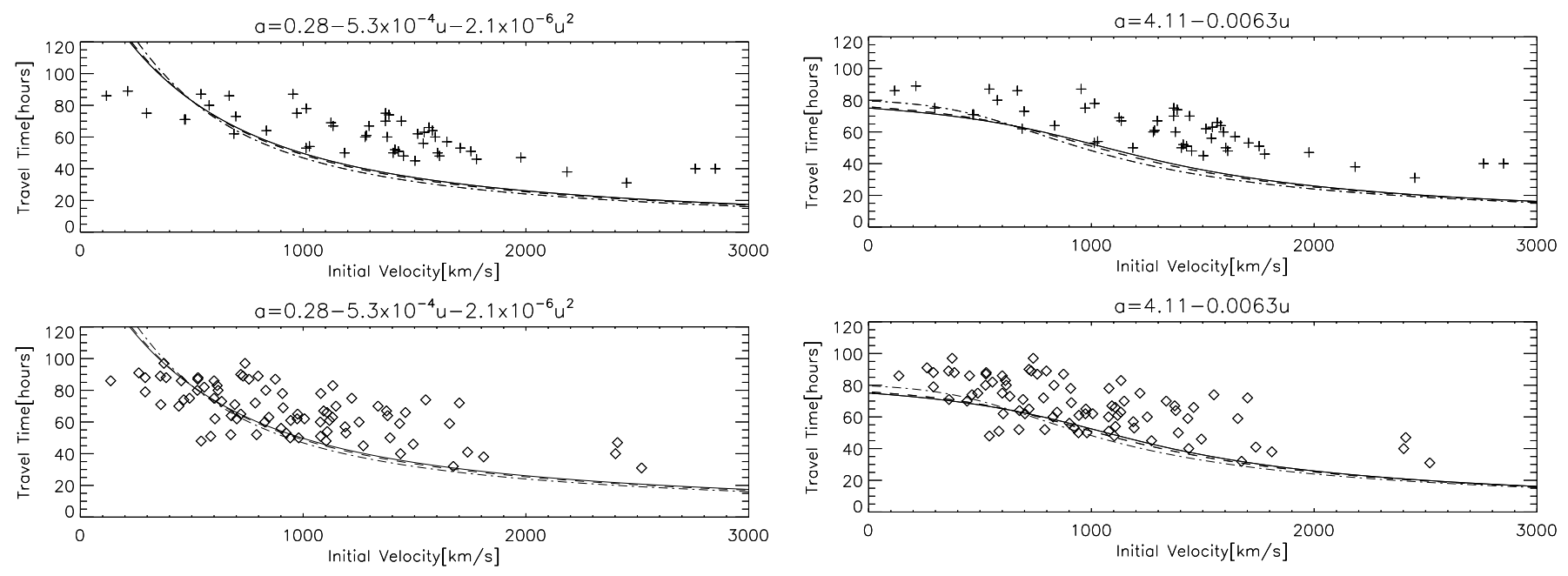

Fig. 3. Comparison between observed and predicted travel time for the model described by the effective acceleration profile $a=0.28-$ $5.3 \times 10^{-4} u-2.1 \times 10^{-6} u^{2}$. The upper panel displays data points for 49 events with corrected velocities and bottom panel for 83 events with uncorrected velocities. The solid, dashed and dot-dashed lines represent model estimations for acceleration-cessation distances equal to $1.0,0.75$ and $0.5 \mathrm{AU}$, respectively.

\subsubsection{Acceleration from extreme points}

From the point of view of interaction with the solar wind, there are three types of CMEs: (i) slow CMEs accelerated by solar wind; (ii) CMEs which have almost the same velocity as the solar wind and (iii) fast CMEs which are decelerated. Before reaching the Earth, some CMEs attain the solar wind speed; for these events, the time over which acceleration takes place may be shorter, but we do not know at what distance the acceleration ceases. The effective acceleration derived by using the 1-AU travel time in this case may not be correct. So we

Fig. 4. Comparison between observed and predicted travel time for the model described by effective acceleration profile $a=4.11-0.0063 u$. The upper panel displays data points for 49 events with corrected velocities and bottom panel for 83 events with uncorrected velocities. The solid, dashed and dot-dashed lines represent model estimations for acceleration-cessation distances equal to 1.0, 0.75 and $0.5 \mathrm{AU}$, respectively.

introduce the effective acceleration from two groups of CMEs only.

We choose CMEs in two extreme cases: i) when there is no acceleration (cessation distance zero) and ii) CMEs which are still decelerating at 1-AU (cessation distance greater than 1-AU). The first condition is fulfilled by CMEs with an initial speed close to the solar wind speed (we assume $\sim 450 \mathrm{~km} \mathrm{~s}^{-1}$ ). The second group consists of the fastest CMEs which at 1-AU have speeds much higher than the solar wind speed ( the three fastest events from our sample CME speed $>2400 \mathrm{~km} \mathrm{~s}^{-1}$ ). We assume that the two sub-samples represent CMEs that do not stop accelerating at any place between the Sun and Earth. 

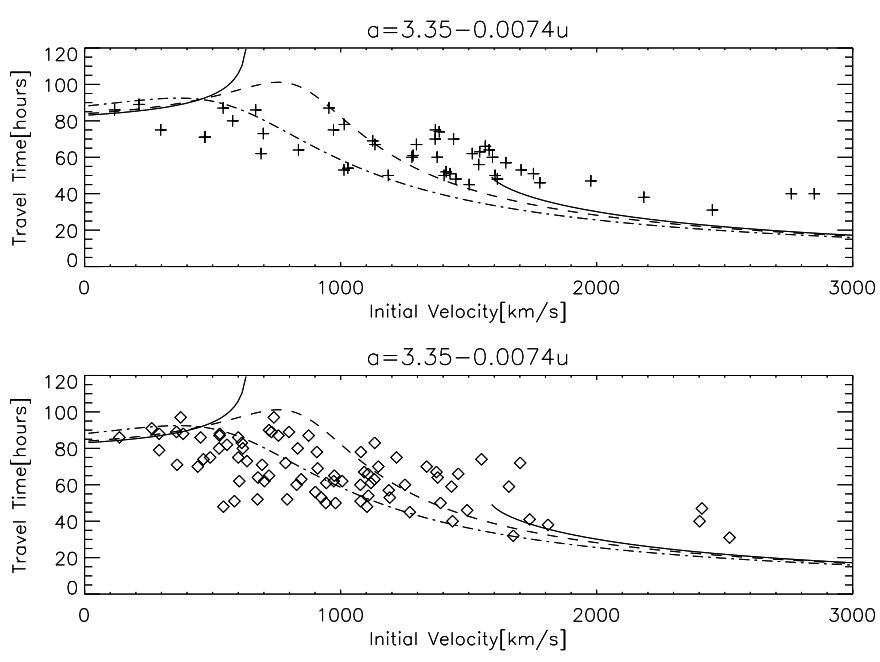

Fig. 5. Comparison between observed and predicted travel time for the model described by effective acceleration profile $a=3.35-0.0074 u$. The upper panel displays data points for 49 events with corrected velocities and bottom panel for 83 events with uncorrected velocities. The solid, dashed and dot-dashed lines represent model estimations for acceleration-cessation distances equal to $1.0,0.75$ and $0.5 \mathrm{AU}$, respectively.

So for these CMEs the 1-AU travel time $(t)$ should be equal to the real acceleration time. Assuming that the acceleration depends linearly on the initial speed, we get an extremum formula for CME acceleration

$a_{\mathrm{ext} 1}=3.35-0.0074 u$.

This relationship is shown by the dot-dashed line in the Fig. 2 (the left panel). As this is a limiting relationship, the remaining data points, not included in the determination of this line, do not fit the line very well. They mostly lie above the line and represent CMEs for which the acceleration may cease at some (unknown) distance between the Sun and Earth.

\subsubsection{1-AU arrival time for the extreme model}

Firstly, we considered the model with arbitrary fixed acceleration cessation distances. The prediction curves, corresponding to the acceleration cessation distances of $0.50,0.75$ and 1-AU, together with both data sets are presented in Fig. 5. The estimated travel times for CMEs with initial velocities in the range $1200-1500 \mathrm{~km} \mathrm{~s}^{-1}$ and an acceleration cessation distance equal to $1-\mathrm{AU}$ is not bounded. Results for the models with acceleration cessation distances equal to 0.75 and $0.50 \mathrm{AU}$ are very similar to those shown by the model presented in Paper I. The slow CMEs seem to be slightly faster and fast CMEs slightly slower than predictions obtained with the model.

\subsubsection{1-AU arrival time for the extreme model and cessation distances dependent on CME corrected velocities}

Finally, we use the extreme model (Eq. (5)) and we assume that the acceleration cessation distance depends on the initial space
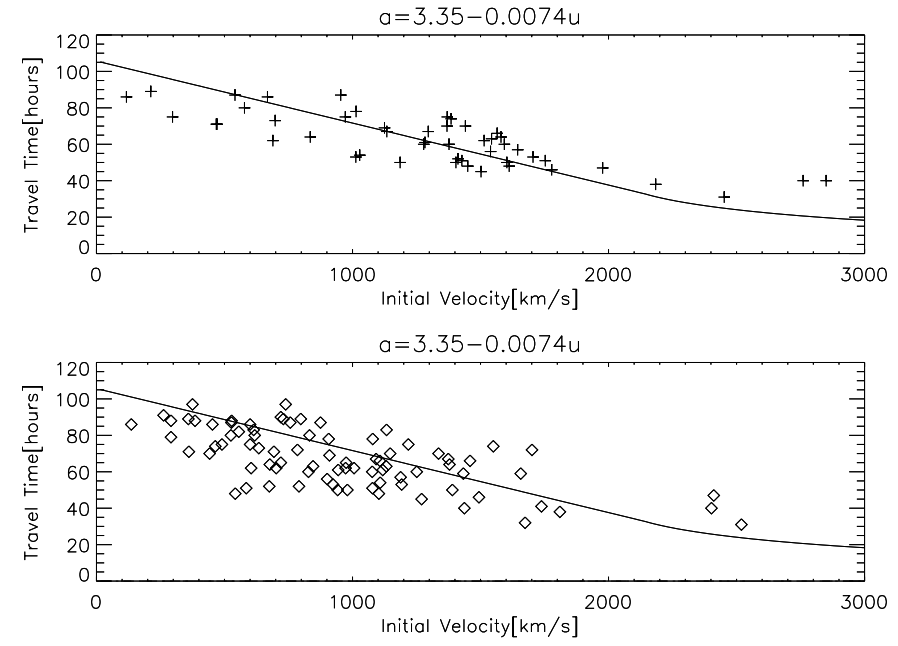

Fig. 6. Comparison between observed and predicted travel time for the model described by effective acceleration profile $a=3.35-0.0074 u$ and acceleration cessation distance dependant on initial velocity of CMEs. The upper panel displays data points for 49 events with corrected velocities and bottom panel for 83 events with uncorrected velocities.

velocity. In this model, we assume that each CME at the beginning phase of propagation is accelerated or decelerated until it achieves a velocity equal to $450 \mathrm{~km} \mathrm{~s}^{-1}$. From this point a given CME propagates with constant velocity. This means that events with different initial velocities will have different acceleration cessation distances. The fastest events can continue to be decelerated until detection in situ and beyond, without cessation of acceleration at any distance from the Sun. The events with an initial velocity equal to $450 \mathrm{~km} \mathrm{~s}^{-1}$ propagate with constant velocity, similar to the average solar wind speed. This model, in that sense, is different from the previous considerations. In the previous models we assumed the same acceleration cessation distance for all CMEs from the entire initial velocity range. In Fig. 6 we compare the prediction curve with the measured travel time for corrected and uncorrected speeds. The predicted curve is almost a straight line with a flattening at the largest initial speeds. In this case, the agreement between the model and data for corrected velocities is very good (upper panel). The results are not so good for events with uncorrected velocities (lower panel). The data points for corrected velocities seem to be slightly shifted towards larger velocities with respect to data points represented by uncorrected velocities. This means that it will be very difficult to build a single general model to predict travel time with good accuracy for both data sets. To improve the results it is necessary to consider a similar model but for uncorrected velocities.

\subsubsection{1-AU arrival time for the extreme model and cessation distances dependent on CME uncorrected velocities}

In the previous subsection we discussed the model which with good accuracy predicts the arrival time for FHCMEs using estimated initial space velocities. Unfortunately, this model is not very useful in practice. Mostly we have only CME speeds 

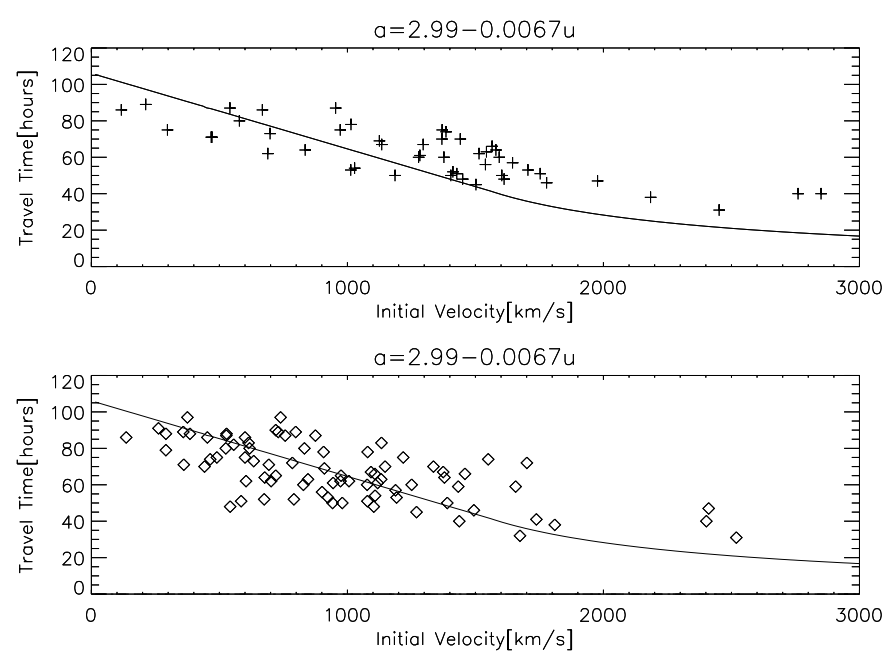

Fig. 7. Comparison between observed and predicted travel time for the model described by effective acceleration profile $a=2.99-0.0067 u$ and acceleration cessation distance dependant on initial velocity of CMEs. The upper panel displays data points for 49 events with corrected velocities and bottom panel for 83 events with uncorrected velocities.

projected to the plane of the sky. We try to build a similar model but using only projected speeds of FHCMEs. Figure 2 (right panel) is a scatter plot of the effective acceleration for the 83 FHCME-ICME pairs obtained using sky plane speeds. As before, we obtained the acceleration profile as

$a_{\mathrm{ex} 2}=2.99-0.0067 u$,

a linear fit to two sub-samples of CMEs, one consisting of three events with projected velocities close to solar wind speed and the other consisting of the three fastest events from the 83 FHCMEs. We considered this acceleration profile assuming that the acceleration cessation distance depends on the velocity of a given CME. It vanishes when a CME reaches the average velocity of the solar wind equal to $450 \mathrm{~km} \mathrm{~s}^{-1}$. Comparison between the observed and predicted travel times for the acceleration in Eq. (6) is presented in Fig. 7 for both data sets. Now, the agreement between the model and data set for uncorrected velocities is very good (bottom panel). Data points for the corrected velocity do not fit this model very well (upper panel). They are mostly scattered above the prediction curve.

\subsection{Distribution of estimated errors}

In Fig. 8, histograms of distribution of estimate errors for models with effective acceleration profiles $a=3.35-0.0074 u$ (Eq. (5)) and $a=2.99-0.0067 u$ (Eq. (6)) are presented. The errors are computed as the deviation of the observed points from the predicted values. In these, we considered an acceleration cessation distance dependent on the initial speeds of the CMEs. Panels 1 and 3 show the distribution of errors for the 49 events with corrected initial velocities and panels 2 and 4 for 83 events with uncorrected initial velocities. For events with corrected initial velocities and the first acceleration profile (Eq. (5)), errors have a Gausian distribution with a peak at $0 \mathrm{~h}$. The mean error of $8.7 \mathrm{~h}$ is lower than the one obtained in Paper I (first panel, Fig. 8). The distribution of errors for the same profile but for events with uncorrected initial velocities has a Gaussian profile but with a peak shifted to $-6 \mathrm{~h}$ (second panel, Fig. 8). The situation changes when we consider the effective acceleration $(a=2.99-0.0067 u)$ from uncorrected speeds. In this case, the error distribution has a Gausian profile with a peak at 0 hours for events with uncorrected initial velocities (fourth panel, Fig. 8). The mean error of $11.2 \mathrm{~h}$ is nearly the same as the one obtained in Paper I (2001a). The error distribution for that profile and corrected velocities is almost flat with two peaks at -12 and $18 \mathrm{~h}$ (third panel, Fig. 8).

\section{Summary}

In this paper we tested different models to predict the $1 \mathrm{AU}$ arrival time of full halo CMEs. Although the models are based on the effective acceleration representing the solar wind-CME interaction, as postulated by Gopalswamy et al. (2000a and 2001a), the present work is different in the following aspects: (1) we consider only full FHCMEs (width $=360^{\circ}$ ); (2) we start from halo CMEs, whereas the previous consideration started with ICMEs; (3) the present sample includes CMEs of wider range of initial velocities; (4) we have a new method to obtain the space-speed of CMEs (Michałek et al. 2003). To improve prediction, we introduced the effective acceleration from two groups of CMEs only. We assume that the two sub-samples represent CMEs which do not have acceleration cessation at any place between the Sun and Earth. In addition, an acceleration cessation distance that is dependent on the initial velocity of a given event was introduced. Using our new method to determine acceleration profiles we were able to predict the arrival time of HCMEs with an average error of 8.7 and $11.2 \mathrm{~h}$ for space and projected initial velocities, respectively. Although we could not reduce the average prediction errors relative to Gopalswamy's et al. (2001a) results, we obtained a Gaussian error distribution with a peak centered at $0.0 \mathrm{~h}$. Our predictions, in comparison to previous results, cover observations in a much larger velocity range. We showed that it is very important to adjust the appropriate acceleration profile to estimate $1 \mathrm{AU}$ arrival time of halo CMEs. We demonstrated that the determination of space velocity is not important to estimate the arrival of CMEs at $1 \mathrm{AU}$. From the inspection of Figs. 6 and 8 it is clear that it will be very difficult to improve the presented model. Only a new set of data (future events) or observation through the entire Sun-Earth distance could allow us to build a new, better model predicting $1 \mathrm{AU}$ arrival time of FHCMEs. Now, in a very simple way, using our fits we can forecast FHCME appearance at the Earth. In the case of the fastest FHCMEs, errors are bigger than 50 percent of the entire travel time. The fastest FHCMEs always arrive always later than the predictions. This could be explained by interactions with earlier slower events. Interactions are most possible in the case of the fastest CMEs. We have to point out some limitations of the models used in this work. (i) The presented models work very well with the full halo CMEs. We must be careful when using this model to predict arrival times for other types of CMEs. For example, partial halo CMEs, which do not hit the magnetosphere head-on, would arrive slightly later than our prediction. As we 

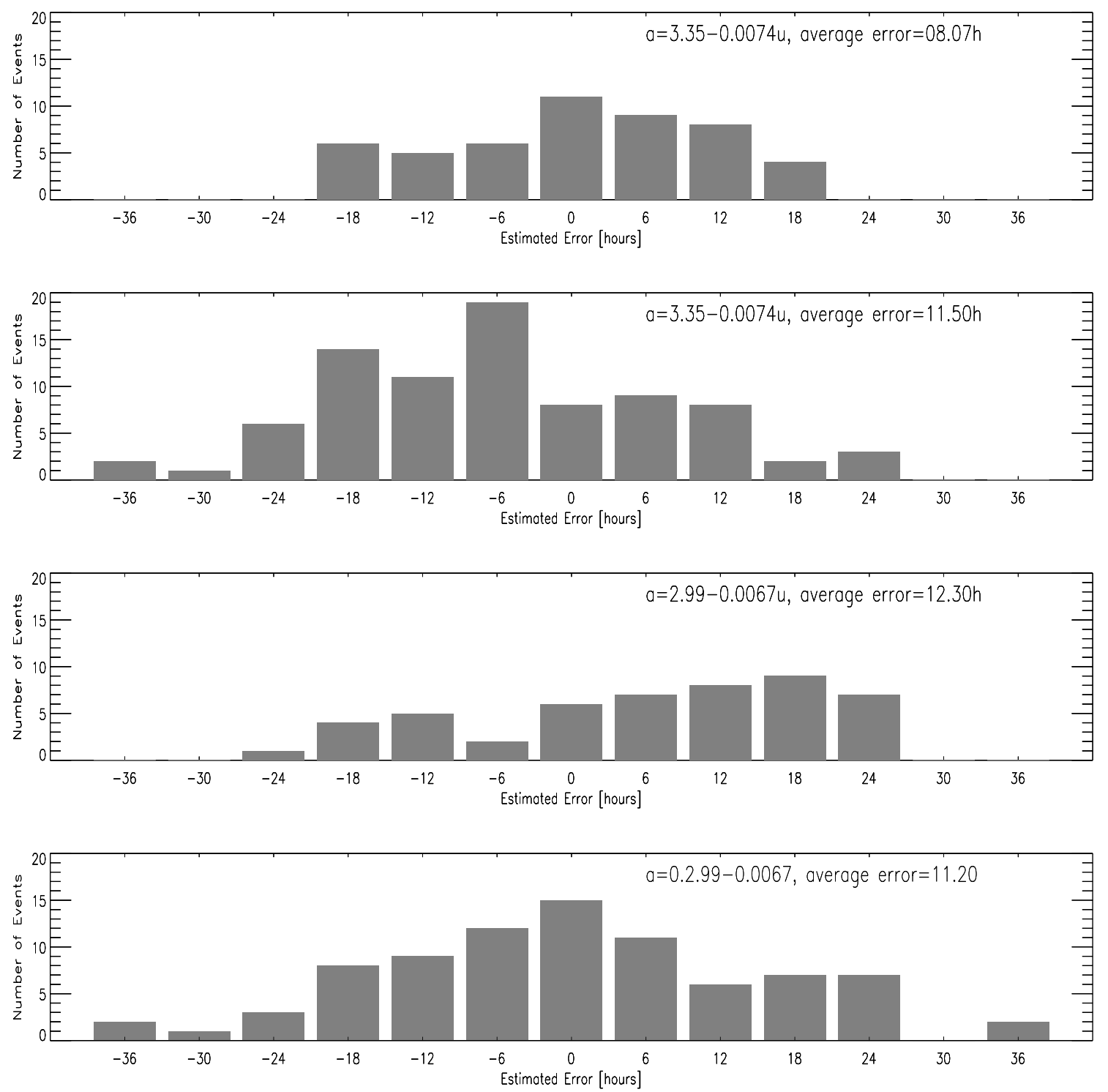

Fig. 8. The histograms illustrate the distribution of estimated errors in the arrival time for models with the effective acceleration profile $a=$ $3.35-0.0074 u$ (two upper panels), $a=2.99-0.0067 u$ (two bottom panels) and the acceleration cessation distance dependant on initial velocities of CMEs. The panels 1 and 3 display distribution of errors for 49 events with corrected velocities and the panels 2 and 4 display distribution of errors for 83 events with uncorrected velocities.

demonstrated, each population of CMEs may need a separate acceleration profile for an accurate prediction. (ii) We use the average effective acceleration depending only on the initial velocity. The real acceleration could change over the entire SunEarth distance. It depends on the nature of the friction and properties of the solar wind.

Acknowledgements. In this paper we used data from $\mathrm{SOHO} /$ LASCO CME catalog at the CDAW Data Center, NASA/GSFC in collaboration with the Naval Research Laboratory and NASA. SOHO is a project of international cooperation between ESA and NASA. Work Supported by NASA/LWS and NSF/SHINE programs.

Work done by Grzegorz Michałek was also supported by Komitet Badań Naukowych through the grant PB 0357/P04/2003/25. AL was partially supported by UNAM grant PAPIIT IN 119402.

\section{References}

Burlaga, L. F., Wang, C., Richardson, J. D., \& Ness, N. F. 1988, J. Geophys. Res., 93, 7217

Burlaga, L. F., Wang, C., Richardson, J. D., \& Ness, N. F. 2002, A\&AS, 34, 752

Burlaga, L. F., Wang, C., Richardson, J. D., Ness, N. F., et al. 2003a, ApJ, 585, 115893

Burlaga, L. F., Berdichevsky, D., Gopalswamy, N., Lepping, R., \& Zurbuchen, T. 2003b, J. Geophys. Res., 108, SSH2-1

Cane, H. V., Richardson, I. G. St., \& Cyr, O. C. 2000, Geophys. Res. Lett., 27, 3591

Cane, H. V., \& Richardson, I. G. 2003, J. Geophys. Res., 108, SSH6-1 Gopalswamy, N., Kaiser, M. L., Lepping, R. P., et al. 1998, J. Geophys. Res., 103, 307

Gopalswamy, N., Lara, A., Lepping, R. P., et al. 2000a, Geophys. Res. Lett., 27, 145

Gopalswamy, N., Kaiser, M. L., Thompson, B. J., et al. 2000b, Geophys. Res. Lett., 27, 1427 
Gopalswamy, N., Lara, A., Yashiro, S., Kaiser, M. L., \& Howard, R. A. 2001a, J. Geophys. Res., 106, 29207

Gopalswamy, N., Yashiro, S., Kaiser, M. L., Howard, R. A., \& Bougeret, J.-L. 2001b, J. Geophys. Res., 106, 29219

Gopalswamy, N., Yashiro, S., Kaiser, M. L., Howard, R. A., \& Bougeret, J.-L. 2001c, ApJ, 548, L91

Gopalswamy, N., Yashiro, S., Michałek, G., et al. 2002, ApJ, 572, L103

Gopalswamy, N. 2003, Proc. ISCS Symposium, Tatranska Lomnica (Noordwijk: ESA Publication Division), 403
Gopalswamy, N., et al. 2003a, Adv. Space. Res., in press

Gopalswamy, N., et al. 2003b, Geophys. Res. Lett., 30, Issue 12, 8015

Lepping, R. P., Burlaga, L. F., \& Jones, J. A. 1990, J. Geophys. Res.

Leblanc, Y., \& Dulk, G. A. 2001, J. Geophys. Res., 106, 25301

Manoharan, P. K., et al. 2004, JGR, in press

Michałek, G., Gopalswamy, N., \& Yashiro, S. 2003, ApJ, 584, 472

Smart, D. F., \& Shea, M. A. 1985, J. Geophys. Res., 90, 183

Smith, Z., \& Dryer, M. 1990, Sol. Phys., 129, 387 


\section{Online Material}


Table 1. The list of HCME-ICME pairs.

\begin{tabular}{|c|c|c|c|c|c|c|}
\hline Date & Time & $\begin{array}{l}\text { Speed } \\
\mathrm{km} \mathrm{s}^{-1}\end{array}$ & $\begin{array}{l}\text { Space velocity } \\
\mathrm{km} \mathrm{s}^{-1}\end{array}$ & $\begin{array}{l}\text { Travel time } \\
\mathrm{h}\end{array}$ & ICME date & $\begin{array}{l}\text { ICME Speed } \\
\mathrm{km} \mathrm{s}^{-1}\end{array}$ \\
\hline $1997 / 01 / 06$ & $15: 10: 42$ & 136 & 117 & 86 & $1997 / 01 / 10$ & 436 \\
\hline 1997/02/07 & $00: 30: 05$ & 490 & 297 & 75 & $1997 / 02 / 10$ & 460 \\
\hline 1997/04/07 & $14: 27: 44$ & 875 & 954 & 87 & $1997 / 04 / 11$ & 470 \\
\hline $1997 / 05 / 12$ & 06:30:09 & 464 & - & 74 & $1997 / 05 / 15$ & 450 \\
\hline $1997 / 09 / 28$ & 01:08:33 & 359 & 212 & 89 & $1997 / 09 / 22$ & 475 \\
\hline 1997/09/17 & $20: 28: 06$ & 375 & - & 97 & $1997 / 09 / 03$ & 425 \\
\hline 1997/11/04 & 06:10:05 & 785 & - & 72 & 1997/11/07 & 440 \\
\hline $1998 / 01 / 21$ & $06: 37: 25$ & 361 & 468 & 71 & $1998 / 01 / 24$ & 400 \\
\hline $1998 / 01 / 25$ & $15: 26: 34$ & 693 & 471 & 71 & $1998 / 01 / 29$ & 390 \\
\hline $1998 / 04 / 29$ & $16: 58: 54$ & 1374 & 1134 & 67 & $1998 / 05 / 02$ & 515 \\
\hline 1998/05/01 & 23:40:09 & 585 & 1427 & 51 & $1998 / 05 / 04$ & 600 \\
\hline $1998 / 05 / 02$ & $05: 31: 56$ & 542 & 1612 & 48 & $1998 / 05 / 04$ & 650 \\
\hline $1998 / 10 / 15$ & 10:04:05 & 262 & - & 91 & $1998 / 10 / 19$ & 383 \\
\hline 1998/11/04 & 07:54:07 & 527 & 541 & 87 & $1998 / 11 / 07$ & 475 \\
\hline $1998 / 11 / 05$ & $20: 44: 59$ & 1118 & 1283 & 61 & $1998 / 11 / 08$ & 620 \\
\hline 1999/04/13 & 03:30:05 & 291 & - & 88 & $1999 / 04 / 13$ & 406 \\
\hline $1999 / 05 / 03$ & $06: 06: 05$ & 1147 & 1369 & 70 & $1999 / 05 / 06$ & 400 \\
\hline $1999 / 06 / 22$ & $18: 54: 05$ & 1133 & - & 83 & $1999 / 06 / 26$ & 350 \\
\hline $1999 / 06 / 24$ & $13: 31: 24$ & 975 & - & 65 & $1999 / 06 / 27$ & 450 \\
\hline $1999 / 06 / 29$ & $07: 31: 26$ & 634 & 698 & 73 & $1999 / 07 / 02$ & 600 \\
\hline $1999 / 09 / 20$ & 06:06:05 & 604 & - & 62 & $1999 / 09 / 20$ & 604 \\
\hline $2000 / 01 / 18$ & $17: 54: 06$ & 739 & - & 97 & $2000 / 01 / 22$ & 400 \\
\hline 2000/02/08 & 09:30:06 & 1079 & - & 78 & $2000 / 02 / 11$ & 425 \\
\hline $2000 / 02 / 09$ & $19: 54: 17$ & 910 & 1125 & 69 & $2000 / 02 / 12$ & 543 \\
\hline $2000 / 02 / 10$ & 02:30:05 & 944 & - & 61 & $2000 / 02 / 12$ & 540 \\
\hline $2000 / 02 / 12$ & $04: 31: 20$ & 1107 & - & 66 & $2000 / 02 / 14$ & 550 \\
\hline $2000 / 02 / 17$ & $20: 06: 05$ & 600 & 668 & 86 & $2000 / 02 / 21$ & 380 \\
\hline $2000 / 04 / 04$ & $16: 32: 37$ & 1188 & 1645 & 57 & $2000 / 04 / 06$ & 570 \\
\hline $2000 / 06 / 02$ & $10: 30: 06$ & 442 & - & 70 & $2000 / 06 / 05$ & 470 \\
\hline 2000/06/06 & $15: 54: 05$ & 1108 & 1028 & 54 & $2000 / 06 / 08$ & 760 \\
\hline 2000/07/07 & $10: 26: 06$ & 453 & - & 86 & $2000 / 07 / 11$ & 440 \\
\hline $2000 / 07 / 11$ & $13: 27: 23$ & 1078 & 1753 & 51 & $2000 / 07 / 13$ & 600 \\
\hline $2000 / 07 / 14$ & $10: 54: 07$ & 1674 & - & 32 & $2000 / 07 / 15$ & 990 \\
\hline $2000 / 07 / 25$ & 03:30:06 & 528 & - & 88 & $2000 / 07 / 28$ & 471 \\
\hline 2000/08/09 & $16: 30: 05$ & 702 & - & 62 & $2000 / 08 / 12$ & 567 \\
\hline 2000/09/12 & $11: 54: 05$ & 1550 & 1385 & 74 & $2000 / 09 / 15$ & 375 \\
\hline $2000 / 09 / 16$ & $05: 18: 14$ & 1251 & 1278 & 60 & $2000 / 09 / 18$ & 760 \\
\hline $2000 / 10 / 02$ & $20: 26: 05$ & 525 & 578 & 80 & $2000 / 10 / 05$ & 510 \\
\hline 2000/10/09 & $23: 50: 05$ & 798 & - & 89 & $2000 / 10 / 13$ & 395 \\
\hline $2000 / 10 / 25$ & $08: 26: 06$ & 757 & - & 87 & $2000 / 10 / 28$ & 375 \\
\hline $2000 / 11 / 03$ & $18: 26: 06$ & 291 & - & 79 & $2000 / 11 / 06$ & 535 \\
\hline 2000/11/08 & $23: 06: 05$ & 1738 & - & 41 & $2000 / 11 / 10$ & 850 \\
\hline $2000 / 11 / 24$ & 05:30:05 & 924 & 1013 & 53 & $2000 / 11 / 26$ & 475 \\
\hline $2000 / 11 / 25$ & $01: 31: 58$ & 2519 & 2452 & 31 & $2000 / 11 / 26$ & 475 \\
\hline $2000 / 11 / 25$ & 09:30:17 & 675 & - & 52 & $2000 / 11 / 27$ & 575 \\
\hline $2000 / 11 / 26$ & 17:06:05 & 980 & 1603 & 50 & $2000 / 11 / 28$ & 590 \\
\hline $2001 / 01 / 05$ & $17: 00: 05$ & 828 & 1593 & 60 & $2001 / 01 / 11$ & 350 \\
\hline 2001/01/10 & $00: 54: 05$ & 832 & - & 80 & $2001 / 01 / 10$ & 420 \\
\hline 2001/03/19 & $05: 26: 06$ & 385 & - & 88 & $2001 / 03 / 22$ & 390 \\
\hline $2001 / 03 / 25$ & $17: 06: 05$ & 677 & 835 & 64 & $2001 / 03 / 28$ & 600 \\
\hline $2001 / 03 / 29$ & $10: 26: 05$ & 942 & 1186 & 50 & $2001 / 03 / 31$ & 650 \\
\hline $2001 / 04 / 05$ & $17: 06: 05$ & 1390 & 1404 & 50 & $2001 / 04 / 08$ & 500 \\
\hline 2001/04/06 & 19:30:02 & 1270 & 1502 & 45 & $2001 / 04 / 08$ & 750 \\
\hline $2001 / 04 / 09$ & $15: 54: 02$ & 1192 & 1705 & 53 & $2001 / 04 / 11$ & 670 \\
\hline $2001 / 04 / 10$ & 05:30:00 & 2411 & 1977 & 47 & $2001 / 04 / 12$ & 700 \\
\hline $2001 / 04 / 11$ & $13: 31: 48$ & 1103 & 1450 & 48 & $2001 / 04 / 13$ & 800 \\
\hline $2001 / 04 / 15$ & $15: 56: 27$ & 1701 & - & 72 & $2001 / 04 / 18$ & 360 \\
\hline $2001 / 06 / 26$ & $12: 30: 05$ & 1006 & 689 & 62 & $2001 / 04 / 29$ & 640 \\
\hline
\end{tabular}


G. Michałek et al.: Arrival Time of HCMEs, Online Material p 3

Table 1. continued.

\begin{tabular}{lllllll}
\hline \hline Date & Time & $\begin{array}{l}\text { Speed } \\
\mathrm{km} \mathrm{s}^{-1}\end{array}$ & $\begin{array}{l}\text { Space velocity } \\
\mathrm{km} \mathrm{s}^{-1}\end{array}$ & $\begin{array}{l}\text { travel time } \\
\mathrm{h}\end{array}$ & ICME date & $\begin{array}{l}\text { ICME speed } \\
\mathrm{km} \mathrm{s}^{-1}\end{array}$ \\
\hline $2001 / 08 / 14$ & $16: 01: 28$ & 618 & - & 80 & $2001 / 08 / 18$ & 550 \\
$2001 / 08 / 25$ & $16: 50: 05$ & 1433 & - & 59 & $2001 / 08 / 28$ & 575 \\
$2001 / 09 / 11$ & $14: 54: 05$ & 791 & 1412 & 52 & $2001 / 09 / 13$ & 390 \\
$2001 / 09 / 24$ & $10: 30: 59$ & 2402 & 2850 & 40 & $2001 / 09 / 26$ & 600 \\
$2001 / 09 / 28$ & $08: 54: 34$ & 846 & - & 63 & $2001 / 09 / 30$ & 530 \\
$2001 / 10 / 09$ & $11: 30: 05$ & 973 & 1514 & 62 & $2001 / 10 / 12$ & 550 \\
$2001 / 10 / 19$ & $16: 50: 05$ & 901 & 1539 & 56 & $2001 / 10 / 22$ & 660 \\
$2001 / 10 / 22$ & $15: 06: 05$ & 1336 & 1441 & 70 & $2001 / 10 / 25$ & 430 \\
$2001 / 10 / 25$ & $15: 26: 05$ & 1092 & 1296 & 67 & $2001 / 10 / 28$ & 460 \\
$2001 / 11 / 04$ & $16: 35: 06$ & 1810 & 2184 & 38 & $2001 / 11 / 06$ & 700 \\
$2001 / 11 / 17$ & $05: 30: 06$ & 1379 & 1580 & 64 & $2001 / 11 / 19$ & 525 \\
$2001 / 11 / 22$ & $23: 30: 05$ & 1437 & 2760 & 40 & $2001 / 11 / 25$ & 730 \\
$2002 / 03 / 15$ & $23: 06: 06$ & 907 & 1014 & 78 & $2002 / 03 / 19$ & 370 \\
$2002 / 04 / 15$ & $03: 50: 05$ & 720 & - & 65 & $2002 / 04 / 17$ & 600 \\
$2002 / 04 / 17$ & $08: 26: 05$ & 1218 & 1370 & 75 & $2002 / 04 / 20$ & 510 \\
$2002 / 05 / 07$ & $04: 06: 05$ & 720 & - & 90 & $2002 / 05 / 10$ & 350 \\
$2002 / 05 / 08$ & $13: 50: 05$ & 614 & - & 83 & $2002 / 05 / 11$ & 500 \\
$2002 / 05 / 16$ & $00: 50: 05$ & 600 & 972 & 75 & $2002 / 05 / 19$ & 450 \\
$2002 / 05 / 22$ & $03: 50: 05$ & 1494 & 1778 & 46 & $2002 / 05 / 23$ & 730 \\
$2002 / 07 / 15$ & $20: 30: 05$ & 1132 & 1543 & 63 & $2002 / 07 / 18$ & 450 \\
$2002 / 07 / 16$ & $15: 06: 08$ & 730 & - & 89 & $2002 / 07 / 20$ & 700 \\
$2002 / 07 / 29$ & $12: 07: 33$ & 556 & - & 82 & $2002 / 08 / 01$ & 454 \\
$2002 / 08 / 16$ & $12: 30: 05$ & 1459 & 1565 & 66 & $2002 / 08 / 19$ & 525 \\
$2002 / 09 / 05$ & $16: 54: 06$ & 1657 & - & 59 & $2002 / 09 / 08$ & 500 \\
$2002 / 11 / 24$ & $20: 30: 05$ & 1077 & 1377 & 60 & $2002 / 11 / 27$ & 550 \\
\hline
\end{tabular}

